\title{
LOCAL STRONG SOLUTIONS \\ OF THE NONHOMOGENEOUS NAVIER-STOKES SYSTEM WITH CONTROL OF THE INTERVAL OF EXISTENCE
}

\author{
Reinhard Farwig - Hermann Sohr - Werner Varnhorn
}

\begin{abstract}
Consider a bounded domain $\Omega \subseteq \mathbb{R}^{3}$ with smooth boundary $\partial \Omega$, a time interval $[0, T), 0<T \leq \infty$, and in $[0, T) \times \Omega$ the nonhomogeneous Navier-Stokes system $u_{t}-\Delta u+u \cdot \nabla u+\nabla p=f,\left.u\right|_{t=0}=v_{0}, \operatorname{div} u=k$, $\left.u\right|_{\partial \Omega}=g$, with sufficiently smooth data $f, v_{0}, k, g$. In this general case there are mainly known two classes of weak solutions, the class of global weak solutions, similar as in the well known case $k=0, g=0$ which need not be unique, see [5], and the class of local very weak solutions, see [1], [2], [3], which are uniquely determined but have no differentiability properties and need not satisfy an energy inequality. Our aim is to introduce the new class of local strong solutions in the usual sense for $k \neq 0, g \neq 0$ satisfying similar regularity and uniqueness properties as in the well known case $k=0, g=0$. Further, we obtain precise information through the given data on the interval of existence $\left[0, T^{*}\right), 0<T^{*} \leq T$. Our proof is essentially based on a detailed analysis of the corresponding linear system.
\end{abstract}

\section{Introduction}

Let $\Omega \subseteq \mathbb{R}^{3}$ be a bounded domain with boundary $\partial \Omega$ of class $C^{2,1}$ and let $[0, T), 0<T \leq \infty$, be the time interval. Then we consider in $[0, T) \times \Omega$ the general nonhomogeneous Navier-Stokes system

$$
u_{t}-\Delta u+u \cdot \nabla u+\nabla p=f,\left.\quad u\right|_{t=0}=v_{0}, \quad \operatorname{div} u=k,\left.\quad u\right|_{\partial \Omega}=g,
$$

2010 Mathematics Subject Classification. 35Q30, 76D05.

Key words and phrases. Instationary Navier-Stokes equations, nonhomogeneous data, strong solutions, weak solutions. 
where the vector $u$ denotes the velocity and $\nabla p$ the associated pressure gradient. In the physical model the divergence $k=\operatorname{div} u$ is assumed to vanish. However, for mathematical reasons it will be convenient, in particular for linear problems, to consider also the more general case of a prescribed divergence $k \neq 0$, compare [4, Remark 1.9(1)]. We refer to [3] and [5] for very weak and weak solutions of this system, respectively. In particular, a counterpart of this paper on the level of very weak solutions can be found in [3], see also [4] for a general review on very weak solutions. However, the focus of this paper is put on the existence of local in time strong solutions.

For simplicity we use for weak and strong solutions the same data class to exploit both theories simultaneously; see [5] for a more general theory of weak solutions.

Next we describe the general assumptions on the data $f, v_{0}, k$ and $g$; here $N(x)$ denotes the outward normal vector at $x \in \partial \Omega$.

\section{Assumptions 1.1 .}

(a) $f=\operatorname{div} F, F \in L^{s / 2}\left(0, T ; L^{q / 2}(\Omega)\right)$, with $4 \leq s \leq 8,4 \leq q \leq 6,2 / s+$ $3 / q=1$,

(b) for $v_{0} \in L_{\sigma}^{2}(\Omega),\left\|v_{0}\right\|_{B_{T}^{q, s}(\Omega)}:=\left(\int_{0}^{T}\left\|e^{-t A} v_{0}\right\|_{q}^{s} d t\right)^{1 / s}<\infty$,

(c) $k \in L^{s}\left(0, T ; L^{q}(\Omega)\right), g \in L^{s}\left(0, T ; W^{-1 / q, q}(\partial \Omega)\right)$ with compatibility condition

$$
\int_{\Omega} k(t) d x=\langle g(t), N\rangle_{\partial \Omega}, \quad t \text {-a.e. }
$$

Here $L^{r}(\Omega)$ denotes the usual Lebesgue space of functions (or vector or matrix fields) with norm $\|\cdot\|_{r}$ and pairing $\langle\cdot, \cdot\rangle_{\Omega}$ with its dual $L^{r^{\prime}}(\Omega), 1<r<\infty, r^{\prime}=$ $r /(r-1)$. Moreover, $L_{\sigma}^{2}(\Omega)={\overline{C_{0, \sigma}^{\infty}(\Omega)}}^{\|\cdot\|_{2}}$ where $C_{0, \sigma}^{\infty}(\Omega):=\left\{v=\left(v_{1}, v_{2}, v_{3}\right) \in\right.$ $\left.C_{0}^{\infty}(\Omega) ; \operatorname{div} v=0\right\}$. Usual Bochner spaces are denoted by $L^{s}\left(0, T ; L^{q}(\Omega)\right)$ with norm $\|\cdot\|_{q, s, T}, 1<q, s<\infty$, and with pairing $\langle\cdot, \cdot\rangle_{\Omega, T}$.

The nonlinear term $u \cdot \nabla u$, defined by $u \cdot \nabla u=\left(u \cdot \nabla u_{1}, u \cdot \nabla u_{2}, u \cdot \nabla u_{3}\right)$, can be written, when $\operatorname{div} u=0$, also as $u \cdot \nabla u=\operatorname{div}(u u)=\nabla \cdot(u u)$ where $u u=$ $\left(u_{i} u_{j}\right)_{i, j=1,2,3}$. Here we note that the divergence of a matrix-valued function $F=\left(F_{i j}\right)_{i, j=1,2,3}$ is defined columnwise.

The initial value norm $\left\|v_{0}\right\|_{B_{T}^{q, s}(\Omega)}$ is a so-called Besov space norm, see [3], [6]-[9], and Section 3 for details. The space $W^{-1 / q, q}(\partial \Omega)$ is a Sobolev trace space of negative order $-1 / q$, namely the dual of the trace space $W^{1 / q, q^{\prime}}(\partial \Omega)$.

Let $P=P_{2}: L^{2}(\Omega) \rightarrow L_{\sigma}^{2}(\Omega)$ denote the Helmholtz projection and $A=A_{2}=$ $-P \Delta$ in Assumption 1.1(b) the Stokes operator on $L_{\sigma}^{2}(\Omega)$. Since $A_{2}=A_{q}$ on $C_{0, \sigma}^{\infty}(\Omega)$, we simply write $A$ for any Stokes operator $A_{q}, 1<q<\infty$; by analogy, since $P_{2}=P_{q}$ on $C_{0}^{\infty}(\Omega)$, we also write $P$ for $P_{q}$. 